Revue internationale P.M.E.

Économie et gestion de la petite et moyenne entreprise

Revue

internationale

PME

\title{
Les districts industriels marshalliens en Italie : une analyse quantitative
}

\section{Fabio Sforzi}

Volume 2, numéro 2-3, 1989

URI : https://id.erudit.org/iderudit/1007933ar

DOI : https://doi.org/10.7202/1007933ar

Aller au sommaire du numéro

Éditeur(s)

Presses de l'Université du Québec

ISSN

0776-5436 (imprimé)

1918-9699 (numérique)

Découvrir la revue

Citer cet article

Sforzi, F. (1989). Les districts industriels marshalliens en Italie : une analyse quantitative. Revue internationale P.M.E., 2(2-3), 159-184.

https://doi.org/10.7202/1007933ar
Résumé de l'article

Cette étude traite des districts industriels marshalliens en Italie et du mode par lequel leur identification concrète est possible. Elle utilise pour cette identification diverses techniques quantitatives basées sur les données statistiques les plus récentes. Surtout cette étude présente le district industriel comme un système local d'industrialisation «légère», soutenu par un environnement social particulier et une production spécialisée dans un même secteur, le tout étant bien localisé. 


\title{
Les districts industriels marshalliens en Italie : une analyse quantitative*
}

\author{
Fabio Sforzi“ \\ Université de Florence
}

\begin{abstract}
RÉSUMÉ
Cette étude traite des districts industriels marshalliens en Italie et du mode par lequel leur identification concrète est possible. Elle utilise pour cette identification diverses techniques quantitatives basées sur les données statistiques les plus récentes. Surtout cette étude présente le district industriel comme un système local d'industrialisation "légère", soutenu par un environnement social particulier et une production spécialisée dans un même secteur, le tout étant bien localisé.
\end{abstract}

\begin{abstract}
This paper deals with the Marshallian industrial districts in Italy within the analytical framework of the model of light industrialization distinguishing the Italian economy after the Second World War, the way in which they can be translate into the reality based on a quantitative approach using at the best the available statistical data. Above all, this paper provides the interpretation of the industrial district as "system of interacting localities" amalgamating a community of people and a population of firms - dominated by a manufacturing industry - localized within a spatiallybounded area to which both of them belong.
\end{abstract}

\section{RESUMEN}

Este estudio trata sobre los distritos industriales Marshallianos en Italia y del modo por el cual su identificaciòn concreta es posible. Utilisa asi para esta identificacion diversas teorias cuantitativas que se basan sobre los datos estadisticos mas recientes. Antetodo, este articulo presenta el distrito industrial, como un sistema local de industrializaciòn «ligera", sotenido por un medio ambiente social particular y una producciòn especializada en un mismo sector, todo lo cual estando bien localizado.

- Cet article est une nouvelle version de l'étude parue dans G. Becattini, (éd), Mercato e forze locali: il distretto industriale, Il Mulino, Bologne, 1987.

* Fabio Sforzi est chercheur invité à l'Institut régional pour la programmation économique de la Toscane et Maître de conférence en Economie régionale au Département d'Economie, Université de Florence. Adresse: I.R.P.E.T. et AISRE - Via la Farina 27/29 50132 FIRENZE. 


\section{Introduction}

La connaissance actuelle de cette réalité concrète que sont les districts industriels s'explique par de longues observations de la part d'économistes et de géographes. Cette exigence dérivait de la nécessité de sortir des premières analyses enthousiastes dans lesquelles, parfois, les districts industriels, comme entités réelles, avaient été utilisés au cours des débats sur le développement économique italien, en particulier dans le cadre de la configuration spatiale du modèle d'industrialisation fondé sur les industries légères.

Ainsi le modèle dominant de développement économique italien basé sur la division Nord-Sud ou sur la comparaison entre le Triangle Industriel et le reste de l'Italie a été remplacé par de nouveaux schèmes d'interprétation, soit le modèle de la Troisième Italie (Bagnasco, 1977) et le modèle NEC (Nord-Est-Centre : Fuà, 1983). Si ces modèles semblent adéquats sur le plan de l'explication économicosociale, ils sont critiquables à partir de l'articulation territoriale de l'espace économique italien qu'ils proposent. En effet, la prise en compte de la dimension locale du développement économique - qui, dans le cas de l'industrialisation légère, peut s'identifier aux systèmes locaux constitués de petites entreprises interdépendantes, spécialisées selon les phases d'un même processus de production et soutenus par un environnement social et institutionnel propre - trouve une correspondance générique dans la configuration territoriale qui accompagne les deux modèles discutés. Ces modèles qui divisent les grandes régions - celle de l'Italie nord-occidentale, celle de l'Italie centre-nord-orientale et celle de l'Italie méridionale - finissent par apparaître uniformément marqués par les processus d'industrialisation, ou encore par l'absence virtuelle de ce processus, lesquels, au lieu d'en distinguer l'échelle selon chaque système local, se réfèrent plutôt aux relations internes et externes qui se sont développées parmi eux.

Cette définition insuffisante de l'organisation territoriale du développement industriel italien des récentes années est accompagnée d'une littérature opposant principalement les prestations économiques de la grande entreprise à celle des petites entreprises manufacturières; ce qui simplifie beaucoup la dimension territoriale de référence et présente les différences en terme de secteurs productifs et de dimensions des entreprises ${ }^{1}$.

(1) À propos de la population des entreprises du district industriel, «c'est une erreur de confondre dans les analyses statistiques ou politico-économiques les petites entreprises des districts industriels avec les petites entreprises opérant dans d'autres contextes. Une telle confusion fait en sorte que ces «petites entreprises» dans les débats ou dans les discussions courantes, apparaissent en bonne partie comme «une boîte vide» (G. Becattini 1989). De façon analogue, confondre la grande entreprise intégrée verticalement à la petite entreprise spécialisée dans une phase d'un processus productif déterminé n'a pas plus de sens. Ce qui a du sens est la confrontation entre deux processus intégrés équivalents, comme, par exemple un processus profitant des avantages de l'économie in- 
Dans ce contexte, on interprète souvent le district industriel comme une catégorie analytique privée d'une échelle territoriale propre. Ainsi, certains l'ont considéré comme faisant partie d'une localité, et même d'une ville particulière; mais d'autres l'ont placé dans un quartier industriel d'une zone métropolitaine ou encore, ont argumenté qu'il ne devait pas être vu comme une entité territorialement définie, puisque les interdépendances entre les entreprises participant au même processus de production peuvent déborder de la localité et s'étendre sur l'aire d'une région sinon de tout le pays ${ }^{2}$.

\section{Le district industriel marshallien}

En Italie, le concept des districts industriels est formulé et présenté comme une catégorie d'analyses économiques alternative au secteur industriel et à l'entreprise, ainsi qu'aux états définis de façon inéquivoque comme concept socioterritorial et économique (Becattini, 1979). En d'autres mots, l'espace fait partie de sa définition propre, de même façon que l'industrie d'un district, constitué d'un système d'éléments de parties intégrés et du district même, est un amalgame d'entreprises et de personnes ${ }^{3}$.

Ces districts représentent un ensemble d'entreprises - de petites dimensions et qui constituent des parties interdépendantes d'un même processus de production - qui interagit comme une communauté de personnes. Cette structure sociale est dominée par des petits entrepreneurs et des travailleurs de l'industrie à l'intérieur d'une zone territoriellement délimitée à laquelle ils appartiennent. Ainsi, la territorialité du district n'est autre qu'un espace localisé de relations fonctionnelles qui s'instaurent entre la population d'entreprises et la communauté d'habitants et qui délimitent un système territorial d'échanges entre eux.

Le district a sa propre échelle territoriale, définie par le système d'interdépendances entre les entreprises réunies, où se trouve la communauté qui regroupe la population qui y vit, et où sont implantées les entreprises locales. C'est, par conséquent, un système localisé - c'est-à-dire réunissant des individus sur un lieu

terne (une seule grande entreprise intégrée verticalement), et un autre profitant des avantages de l'économie externe (un district industriel basé sur un système d'entreprises interagissant et spécialisées dans les différentes phases d'un même processus de production).

(2) Sur cette aspect du débat relatif au district industriel et à ses caractéristiques de processus de spécialisation flexible, ou de désintégration verticale, voir A. Amin, et K. Robins, «Industrial districts and regional development. Limits and possibilities» CURDS, Université de Newcastle upon Tyne.

(3) Une définition plus récente et plus compréhensible du concept de district industriel a été développée en particulier par G. Becattini (1989). 
de travail et de résidence - nouant diverses relations entre eux et impliquant un niveau élevé d'interdépendances; il correspond à la base logique d'un marché local de travail, d'une zone ne comprenant aucune localité distincte, d'un certain niveau de concentration de postes de travail offrant à la majeure partie de la population résidente du travail ou diverses possibilités de changer d'emploi sans changer de lieu de résidence. La principale caractéristique d'un tel marché local de travail est sa délimitation territoriale; ce marché est relativement auto-continu dans le sens où l'offre et la demande du travail tendent à l'équilibre dans la zone sur lequel il s'étend, puisque la majeure partie de la population qui y réside travaille à l'intérieur de cette zone et ceux qui donnent du travail recherchent plus de travailleurs que la localité peut en fournir (Sforzi, 1987).

Ce système d'interactions territorialement localisées correspond à un marché de travail touchant aussi la dimension spatio-temporelle de la vie quotidienne pour la population résidente; cette dimension constitue l'aire dans lequel se passe la majeure partie des relations sociales et économiques. Il est vrai qu'actuellement, dans le système capitaliste, les systèmes d'interactions tendent à s'étendre au-delà de l'espace et à relever de contrats au-delà du temps (Jonas, 1988). Néanmoins les questions relatives à la vie quotidienne sont encore territorialement délimitées; les autres questions plus occasionnelles peuvent être vues comme externes à l'espace local et sont liées àl'environnement avec lequel le système échange de l'information, des biens et des personnes à travers ses frontières. L'environnement est alors vu comme général et s'étendant au reste du monde.

Marshall, discutant des différentes organisations industrielles, a introduit une distinction entre les économies internes et celles externes; il a montré que ces dernières «pouvaient souvent être obtenues par la concentration de plusieurs petites entreprises de même types dans des localités spécifiques ou, comme on dit régulièrement, par la localisation de l'industrie» (Marshall, 1920). Cette «localisation particulière» n'est autre que les districts industriels; ceux-ci peuvent convenablement être vus comme des systèmes locaux dotés d'une propriété constitutive sociale, économique et territoriale qui les différencie des autres systèmes locaux comme ceux dominés par une grande entreprise. La "company-towns», par exemple, se distingue des districts industriels, selon Marshall, par une organisation industrielle et une division du travail différentes.

Le district industriel marshallien se reflète dans une structure sociale qui se caractérise particulièrement par un nombre relativement élevé de personnes qui travaillent pour leur compte et par des règles économiques touchant la famille non seulement en tant que consommatrice mais aussi en tant que productrice. 


\section{$3 \quad$ L'identification du district industriel marshallien dans la réalité concrète}

L'identification du district industriel dans la réalité concrète doit inclure une étude de son évolution. Cette étude permet d'en spécifier le cheminement et peut être expliquée, de façon opérationnelle, comme le jeu d'interactions internes d'un système d'entreprises (de petites tailles) qui seraient concentrées spatialement, se partageraient les diverses phases d'un même processus de productions et qui seraient liées à une population spécifique et isolée (avec les installations industrielles et les résidences sur le même territoire).

Cette définition opérationnelle permet d'établir les traits essentiels d'un processus de recherche ayant pour but de reconnaître un district industriel d'un système d'installations humaines pouvant varier selon la région où celle du pays. Cette démarche analytique compte trois phases principales. La première a pour objet de spécifier la configuration spatiale. La deuxième doit permettre d'établir si une concentration territoriale d'entreprise constitue une zone manufacturière spécialisée. La troisième montrera si, au contraire, un système local est socialement et économiquement caractérisé pour représenter une approximation suffisamment empirique du concept de district industriel.

Dans un premier temps, nous avons réalisé nos analyses à un niveau territorial très détaillé, dans le but de montrer comment résoudre empiriquement la liaison conceptuelle existante entre la notion du système local et celle du district industriel. Par la suite, nous avons étendu notre étude à tout le territoire italien à partir de la configuration générale de son espace économique, et nous avons interprété cette étude en fonction des types de systèmes locaux.

\subsection{Le modèle spatial du système productif}

La première étape consistait à clarifier l'aire de l'étude selon la caractéristique de l'unité productive et sa localisation dans le but d'identifier la forme spatiale de la distribution et de l'interdépendance de l'activité productive ainsi que son espace de spécialisation en émergence. La recherche de l'information et des données provenait du recensement industriel, lequel offrait un nombre élevé de variables capables de décrire le mieux possible les caractéristiques productives tant de la région que de tout le pays. Cette disponibilité des données individuelles - pour chaque unité productive et au niveau des sections de recensement permettant de décrire de façon fine le territoire - a permis d'éliminer les erreurs qui inévitablement peuvent se trouver en utilisant des données statistiques au niveau des seules communes. En obtenant de telles variables au niveau des sections de recensements - méthode efficace pour utiliser la technique de l'analyse multivariée (Openshaw et Sforzi, 1983) - on a pu définir un modèle spatial du système productif sous forme d'espaces générés par les caractéristiques de chacune des petites unités qui constituent les zones 
économiques des territoires étudiés, en combinant l'activité directe ou indirecte spécifique à chacune d'elles ${ }^{4}$.

A titre d'exemple, la figure 1 montre les résultats appliqués à la région de la Toscane comprise entre Florence et Prato. Comme on peut le noter en parcourant la liste des variables, nous n'avons pas pris en compte les variables reliées aux aspects de taille des entreprises, appliquées à une seule unité de production. C'est pourquoi, après cette première analyse, on peut constater qu'il n'y avait pas moyen de discriminer la taille des territoires selon la même spécialisation de production à des fins de configuration spatiale. Toutefois, en tenant compte successivement du système d'entreprises et de leur taille «modeste», nous avons pu compléter la description de chaque zone de spécialisation sur la base de ces caractéristiques.

Pour les régions développées comprenant des districts industriels, on peut utiliser l'exemple de la zone textile caractérisant le district autour de Prato pour y trouver des unités de production où les données peuvent facilement s'appliquer. On peut toutefois se demander, suite à cette discussion de procédure dans l'analyse, si on parle d'une zone spécifique appelée «district du textile de Prato». La réponse,

(4) Cette application réalisée en référence à la Toscane provient de l'utilisation de données d'environ 13000 sections de recensement sur la base d'un ensemble de 59 variables dérivées du recensement général de l'industrie, du commerce, des services et de l'artisanat (Sforzi, Openshaw et Wymer, 1984). Ces variables exprimées sous forme de ratios en pourcentage entre chaque activité productive et l'ensemble des activités productives sont les suivantes : (1) activités connexes à l'agriculture; (2) industrie énergétique; (3) énergie électrique, gaz et eau; (4) extraction des minéraux; (5) métallurgie; (6) matériaux de construction; (7) travail de la pierre et des produits non minéraux; (8) industrie du verre; (9) produits en métal; (13) mécanique; (14) appareils de bureau et de traitement de données; (15) électromécanique; (16) matériel de transport; (17) mécanique de précision; (18) industrie alimentaire; (19) industrie textile; (20) tannerie; (21) production d'articles en cuir; (22) industrie de la chaussure; (23) industrie de l'habillement; (24) industrie du bois et des meubles en bois; (25) industrie de papier; (26) imprimerie et édition; (27) industrie du caoutchouc et des matières plastiques; (28) orfèvrerie; (29) autres industries manufacturières; (30) construction et travaux de voirie; (31) commerce de gros; (32) distribution; (33) commerce de détail dans l'alimentation; (34) commerce de détail des articles sanitaires et de la pharmacie; (35) commerce de détail des articles de l'habillement; (36) commerce de détail des articles d'équipements et pour la maison; (37) commerce de détail des véhicules; (38) commerce de détail du carburant; (39) commerce de détail des livres et des articles et fournitures de bureau; (40) commerce de détail d'articles variés; (41) hôtellerie; (42) restauration; (43) réparation des véhicules et des biens de consommation; (44) livraison et activités connexes; (45) transport; (46) communications; (47) agences de voyage; (48) instituts de crédit et d'assurances; (49) agences immobilières; (50) service d'impression; (51) location des biens mobiliers; (52) administration publique; (53) services d'hygiène publique; (54) éducation; (55) recherche et développement; (56) services sociaux et sanitaires; (57) organisations d'associations professionnelles; (58) services récréatifs et culturels; (59) services personnels. 
Fig. 1 Modèle spatial du système productif
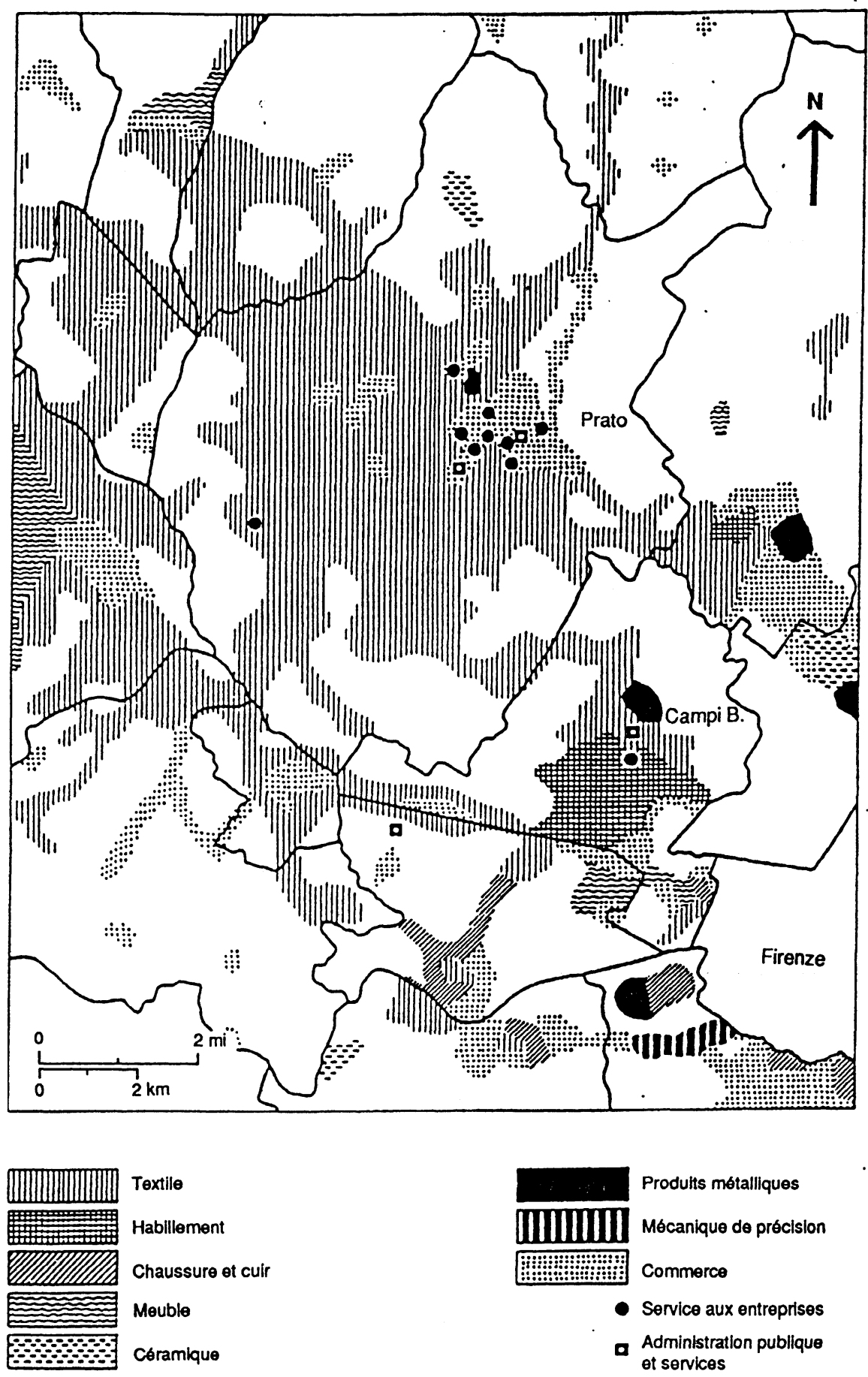
selon la définition des districts industriels, est naturellement négative. Mais en comparant notre étude à d'autres sur les systèmes de production locales, le niveau de détails territoriaux déjà obtenus et la caractérisation productive déjà précisée nous permettent d'être satisfaits des résultats obtenus. Dans une analyse fermée et en connaissant la forme productive qui permet de distinguer les modes de productions et le type de divisions de travail dans la zone de Prato, il serait difficile de démontrer l'existence d'une espèce particulière localisée de relations industrielles et de développement de type autocentré. Mais la définition des districts industriels utilisée ne peut pas être comparée à celle des autres études empiriques puisque nous n'avons pas pris en compte le problème de la structure sociale de cette zone. C'est pourquoi d'autres analyses, pour classifier les zones de spécialisation productive, retiennent ce dernier aspect comme intrinsèquement nécessaire à la compréhension de la nature des systèmes productifs locaux (Garofoli, 1983). Cet aspect a donc dû être pris en compte dans la recherche.

\subsection{Le modèle spatial du système social}

Cette seconde phase reprend la procédure analytique de la phase précédente en ajoutant aux territoires déjà classifiés les caractéristiques sociales et démographiques de la population qui y vit; ceci dans le but d'identifier la forme spatiale tenant compte des zones résidentielles qui permettent de distinguer les groupes sociaux ${ }^{5}$.

(5) Encore dans cet exemple de la Toscane, nous avons utilisé un ensemble de 61 variables au niveau des sections des recensements dont les données ont été reliées à l'unité productive (Sforzi, Openshaw et Wymer 1983). Ces variables, toujours exprimées sous forme de ratio en pourcentage entre les données obtenues et la «population» de référence, sont les suivantes : A) 1. entrepreneurs, dirigeants et professions libérales, 2. employés, 3. travailleurs à leur propre compte, 4. opérateurs apprentis, travailleurs à domicile; B) travailleurs profesionnels, 5. dans l'agriculture, 6. dans l'industrie, 7. dans la construction, 8. dans le commerce, 9. dans les services, 10. dans l'administration publique; C) travailleurs professionnels ayant: 11 . un diplôme de l'école secondaire supérieure, 12 . un diplôme de l'école secondaire, 13. un diplôme élémentaire, 14. sans titre de diplôme; D) données relatives aux conditions professionnelles : 15. 1'âge, 16. les 14-24 ans professionnels, 17. les 14-24 ans aux études, 18. les 14-24 ans en recherche d'un premier emploi, 19. les retraités; E) l'âge de la population : 20 . moins de 5 ans, 21 . les 6-13 ans, 22. les 1418 ans, 23. les 19-24 ans, 24. les 24-44 ans, 25 . les $45-59$ ans, 26 . les 60 ans et plus, 27. la population de passage, 28 . les étrangers; F) la famille composée : 29. d'une personne seule, 30. d'un seul parent avec un ou plusieurs enfants, 31 . de six personnes ou plus, 32. de deux personnes ayant entre 19 et 59 ans, 33. de trois enfants ou plus, 34. de six personnes ou plus, dont deux ont plus de 60 ans, 35 . de deux personnes de plus de 60 ans, 36. de trois à cinq personnes, avec un ou plus ayant 60 ans ou plus; $G$ ) bâtiment où cohabite :37. une ou plusieurs familles, 38 . non occupé, 39 . libre et disponible pour la vente ou la location, 40 . libre ou utilisée comme résidence secondaire, 41 . libre pour d'autres motifs; H) habitations occupées comme : 42 . propriété, usufruit ou rachat, 43. à louer, 44 . à usage gratuit, 45 . avec une ou deux pièces, 46 . avec six pièces ou plus, 47 . avec $\geq 1,5$ personnes par pièce, 48 . avec $\leq 0,5$ personne par pièce, 49 . avec bain et salle de toilette, 50 . sans bain, 51. sans salle de toilette à l'intérieur de l'habitation, 52. avec salle de toilette en commun 
Un exemple des résultats obtenus à la suite de cette phase (toujours relatif à cette partie de la Toscane déjà considérée) peut être vu à la figure 2 (p. 168). Ce type d'analyse, ajoutant l'aspect de structure sociale des espaces résidentiels régionaux, permet de définir un modèle spatial du système social; ce modèle définit une typologie sociale d'espaces localisés qui spécifie la zone de spécialisation de la production déjà délimitée et vérifie la correspondance entre l'identité productive et l'identité sociale.

Le caractère «typique» de la société locale que forme la zone textile de Prato peut apparaître évident, si l'on tient compte des principales caractéristiques sociales comme la présence de classes de travailleurs et de petits entrepreneurs de l'industrie, ou plus particulièrement, de la présence importante d'une main-d'œuvre féminine dans les usines. Cette présence constitue une caractéristique sociale exclusive distinguant cette industrie du reste de la région.

Les études sur le développement économique de la Toscane, comme pour d'autres parties de l'Italie, montrent que l'industrialisation légère a dominé en tout ou en partie le processus d'évolution de la réalité locale. Elle a transformé les autres aspects sociaux particuliers qui l'ont accompagnée. Comme on le verra, elle a soutenu une structure familiale liée aux prestations de travail de ses membres dans la manufacture, et les opérations entrepreneuriales exercées par le chef de famille. Cette implication a nécessairement produit une économie familiale qui crée un phénomène se rencontrant encore dans les petites unités productives mais qui dépasse le noyau familial (IRPET 1975).

Arrivé à ce point de la recherche, on constate qu'on a pu vérifier, selon une interdépendance qui apparaissait de prime abord difficile à établir, «une congruence» entre le milieu social et la forme productive adoptée par le système d'entreprises à la base de la zone de la spécialisation industrielle (Becattini, 1978). En fait, on a pu ainsi confirmer à l'aide d'une procédure objective ce que d'autres analyses avaient trouvé, soit l'existence sur un territoire donné d'un système d'entreprises spatialement concentré, spécialisé dans un certain secteur de l'industrie manufacturière (telle l'industrie textile, comme dans notre exemple) et une population avec une forte cohésion sociale. Cette cohésion repose sur une continuité entre les prestations du travail familial, des travailleurs dépendants et du travail à son propre compte. Ceci a permis de définir cette configuration spatiale comme une entité dans laquelle les processus de socialisation, des valeurs et les comportements sont orientés par la présence d'une industrie qui marque profondément le territoire et représente le centre vers lequel converge les parties représentant les intérêts de la communauté. Tout ceci évoque le processus de transmission culturelle à la base de ce que Marshall dénomme

situé à l'extérieur de l'habitation, 53. avec salle de toilette sans eau courante, 54 . avec chauffage central, 55. avec chauffage autonome, 56. avec chauffage partiel, 57. sans chauffage, 58. sans eau courante à l'intérieur de la maison, 59. sans eau potable de l'aqueduc, 60. sans électricité, 61. autres types de logements. 
Fig. 2 Modèle spatial du système social
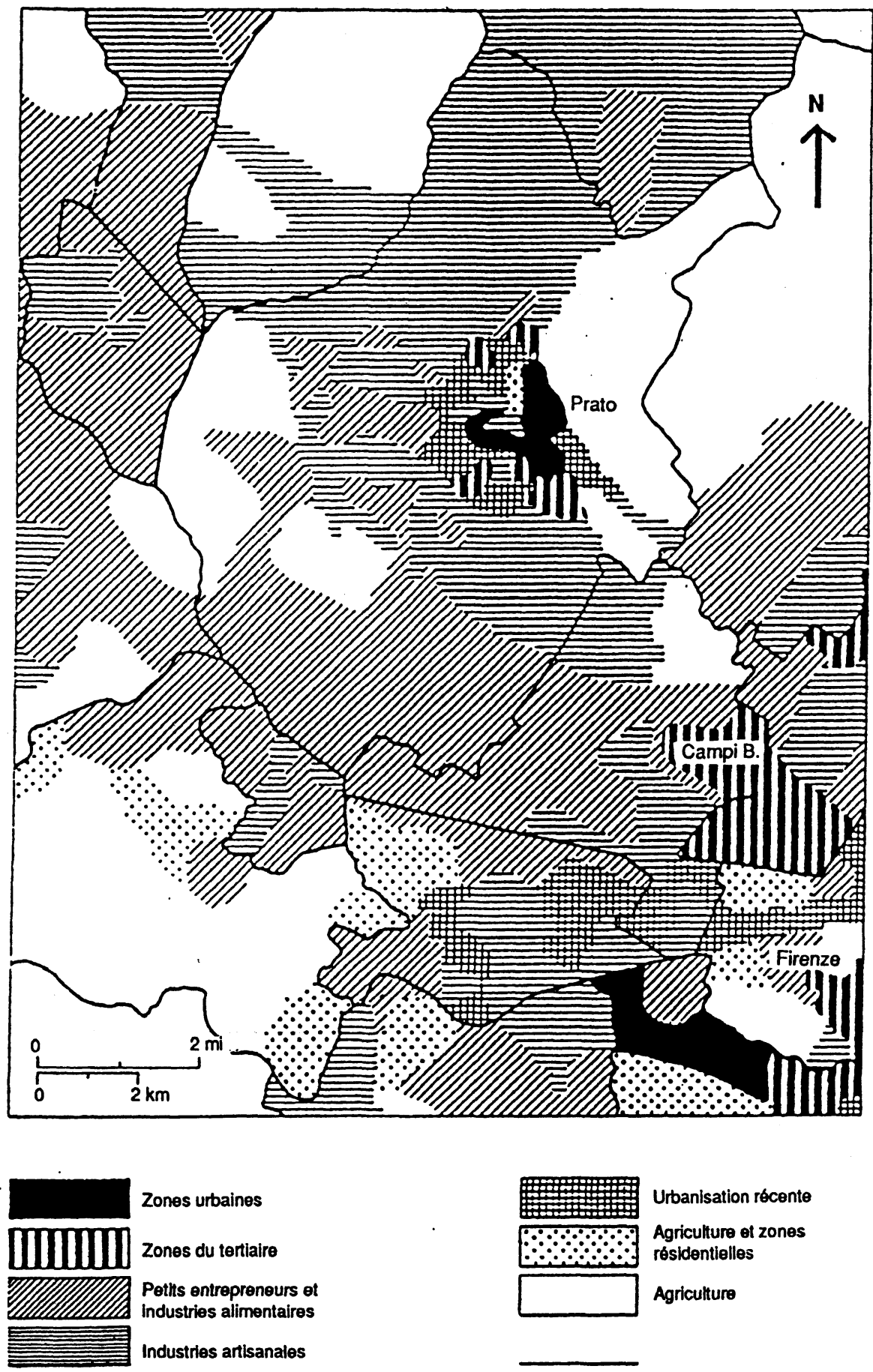
comme l'expression «d'une atmosphère industrielle» qui se développe entre un district industriel et un point de convergence créé par la présence et l'exigence de l'industrie. Ce processus donne une importance majeure à une communauté de personnes cohabitant sur un territoire limité et dans un espace à concentration industrielle naturelle et durable. Mais il faut ajouter un troisième élément conceptuel qui complète cette délimitation spatiale du district industriel.

\subsection{Le modèle spatial du système territorial}

Pour cela, il faut, dans notre démarche de recherche, pouvoir expliquer l'expression «relativement restrictive» expliquant l'origine spatiale du système d'entreprises et de la communauté de personnes; ceci dans le but de compléter la définition du district industriel. Entre les différentes significations que nous avons essayé de donner à cette expression, on peut en retenir une plus appropriée dans le raisonnement conduisant au concept «d'autocontenimento» par lequel les analyses géographiques italiennes définissent la capacité d'un système territorial à intégrer le plus possible les interactions entre les éléments relevant de la localisation résidentielle, de la localisation productive et de la concurrence dans ce mode de reconnaissance propre. Cette mise en question a pour but d'identifier les systèmes territoriaux selon différents niveaux d'ouverture vers l'extérieur (dans un système ouvert, le changement provient de l'échange d'information ou «d'énergie» avec l'environnement qui l'entoure et le contient); mais ceci vaut aussi pour les caractéristiques analogues de comportement des composantes de ces systèmes avec le sur-système qui les entourent.

En effet, on sait qu'un système territorial peut interagir avec d'autres systèmes à travers un nombre relativement faible d'interactions; il se comporte alors comme une entité territoriale. Par contre, si les interactions sont relativement élevées pour maintenir sa propre individualité, le système doit adopter une configuration d'ordre inférieur (Harvey, 1969). Sur la base de ces considérations, et en utilisant comme variable les comportements de la population selon la localisation de leur résidence et de leur travail, on peut générer un réseau d'interaction spatiale entre les installations humaines d'un territoire. Ceci nous permettra de reproduire la réalité selon l'occupation dans un modèle spatial du système territorial pour vérifier l'existence et la configuration d'une organisation locale possédant la propriété des systèmes territoriaux (Chapman, 1977).

Une telle méthode d'analyse quantitative nous a permis de trouver des résultats assez satisfaisants pour identifier les systèmes territoriaux (Sforzi, Openshaw et Wymer, 1982). En utilisant un algorythme pour la régionalisation fonctionnelle (Sforzi, 1982), notre analyse permet de préciser le complexe d'interactions entre la localisation de la commune et les déplacements des journaliers pour leur travail afin d'identifier les systèmes locaux dans les zones de la région. À la figure 3 (p. 170), on peut voir un exemple des résultats obtenus, toujours dans la région de la Toscane. 
Fig. 3 Modèle spatial du système territorial

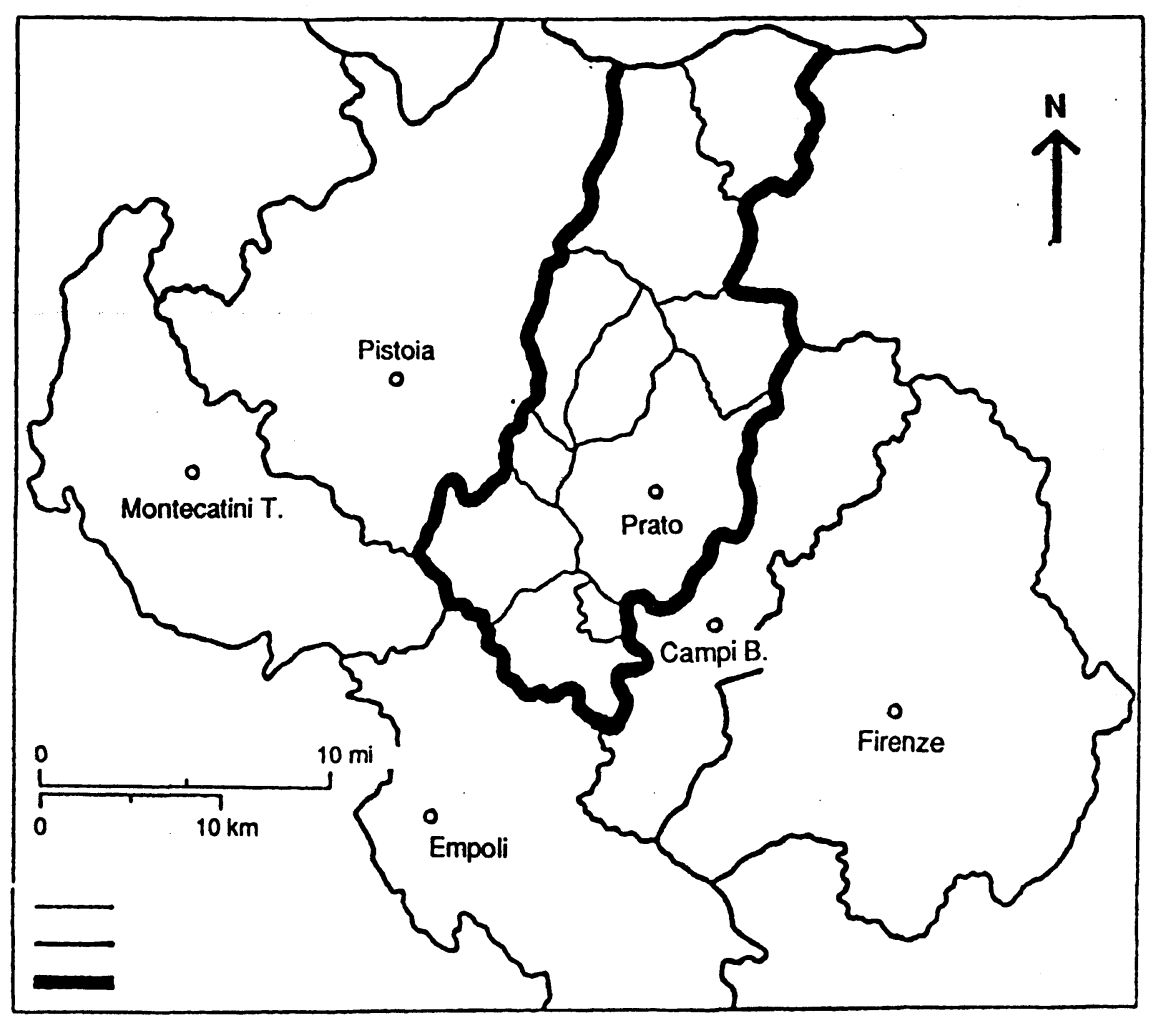

1 Limite des communes

2 Limite du système local

3 Limite du système local de Prato 
Comme il apparaît dans cette figure, le territoire d'installation du système d'entreprises et de la communauté d'habitants délimite le système local ${ }^{6}$ et représente des territoires communs et relativement restreints. On peut y trouver une contiguité entre les populations et les entreprises qui ne se réduit pas à la seule période des heures de travail, mais qui s'étend aux relations sociales, se manifeste dans le temps et l'espace d'une journée de travail ainsi que dans l'apport d'autres activités quotidiennes offertes par la «région» (aussi dans le temps et l'espace, à cause de cette proximité entre les lieux de résidence et les lieux du travail) (Hägerstrand, 1970).

Cette démarche de recherche a donc permis de spécifier de façon la plus complète possible l'identité territoriale du district industriel en tenant compte des échanges entre les habitants et les travailleurs.

\section{$4 \quad$ Les districts industriels marshalliens en Italie}

Avec le grand nombre d'études récentes sur l'organisation de l'espace du système économico-social italien, on a la possibilité de répéter pour tout le pays le procédé empirique de recherche développé en référence avec la Toscane, lequel a conduit à la connaissance du système territorial de Prato en tant que district industriel marshallien spécialisé dans le textile. Le but est de repérer et spécifier les districts industriels dans toute l'Italie.

Ces études auxquelles nous nous référons (ISTAT-IRPET, 1986a, 1986b, 1986c) constituent l'aboutissement d'une phase intense de recherches méthodologiques et empiriques portant sur l'identification d'espaces des systèmes locaux (du travail), de zones des spécialisations productives et de zones résidentielles; ces recherches décrivent exhaustivement la structure, en Italie, de ces ensembles humains et de leurs relations fonctionnelles comme modèle d'espaces discuté précédemment. Pour réaliser cette analyse, il faut d'abord délimiter la dimension territoriale de chaque district en tant que configuration générale des systèmes locaux.

D'une part, comme cela a été discuté précédemment, pour reconnaître un système local comme district industriel marshallien, il n'est pas suffisant que ces derniers soient organisés comme une «totalité de parties interagissantes»; il est nécessaire qu'ils aient aussi certaines «caractéristiques productives» de l'entreprise manufacturière localisée, c'est-à-dire que celle-ci soit répartie selon les diverses phases d'un même processus de production.

(6) L'utilisation du terme «système local» au lieu de celui de «marché local du travail» ou du «système urbain des travailleurs» est plus général et permet de mieux comprendre tous les phénomènes inclus. Pour appuyer ces arguments, voir Sforzi (1982) et Sforzi (1987). 
D'autre part, en l'absence d'un modèle capable de délimiter raisonnablement une entité territoriale auto-continue, on ne saurait, grosso modo, ramener à quelques objectifs économiques et géographiques la configuration spatiale de la structure sociale et de la structure productive, avec pour résultat d'élever au rang de «système» regroupé les installations productives et les zones habitées par le seul fait qu'elles soient physiquement contiguës, alors qu'on ne reconnait pas une telle configuration comme ayant un «caractère» de système local propre et des relations spécifiques (Sforzi, 1982).

La configuration générale assumée par les systèmes locaux et reproduite à la figure 4 (p. 174), représente donc, selon notre raisonnement, un élément fondamental pour s'engager et pour parvenir à identifier les districts industriels marshalliens en Italie, selon le processus de recherche déjà expérimenté.

La phase suivante consiste à rechercher la structure sociale de chaque système classifié selon les caractéristiques de la population résidente, dans le but de sélectionner les caractéristiques dominantes et «typiques» des districts industriels marshalliens.

Cette typologie sociale distincte des districts repose sur la présence de petits entrepreneurs et de travailleurs de l'industrie, d'une main-d'oeuvre féminine importante et d'une structure familiale réunissant vieux et jeunes dans le même milieu de travail (figure 5, p. 175). Une rétrospective de cette caractérisation sociale expliquant le processus de développement basé sur l'industrialisation dans les industries «légères» (IRPET, 1969, 1975) n'est cependant plus à faire. Aussi la forme spatiale décrite des systèmes locaux, avec sescaractéristiques structurelles typiques, peut être appliquée à l'échelle nationale, à partir de ce modèle territorial d'industrialisation légère. Encore une fois, elle doit cependant tenir compte des formes de production et d'habitation au niveau local dans le développement économique de la «campagne urbanisée» (IRPET, 1975).

La signification de ces résultats, sur le plan de la réflexion théorique, peut encore suggérer des liaisons évidentes en «amont» ou en «aval» entre le modèle d'industrialisation «légère» et celui de district industriel marshallien. Comparé au premier modèle, celui des districts marshalliens représente une phase de développement ultérieur spécialisé et constitue une «forme de transition» vers un état d'ordre et d'organisation croissante qui differe d'un état interne du système local ou des relations entre les différents systèmes locaux ou industriels et qui conduit vers une industrialisation des fonctions à un niveau d'organisation plus élevé7.

(7) «... d'un côté le développement de l'organisation, sociale ou physique, implique une subdivision dominante des fonctions entre ses diverses parties et, de l'autre, une liaison plus forte entre elles. Chaque partie semble être de moins en moins auto-suffisante, et dépend pour son développement de plus en plus d'autres parties; ainsi tout désordre dans une partie quelconque de l'organisation plus large va affecter aussi les autres parties. Cette croissance dans la subdivision des fonctions, ou dans la «différenciation» comme on l'appelle, se manifeste en regard de l'industrie par rapport à la division du travail, et au 
C'est pourquoi, dans notre intérêt, pour dessiner la carte des districts industriels marshalliens en Italie, il faut mettre l'accent sur une bonne connaissance de la réalité concrète à travers la diffusion de l'identité sociale des systèmes locaux. Ainsi, on parviendra à valider de façon critique les analyses de référence touchant la caractérisation productive de ces systèmes dans la mesure où celle-ci permet de lier l'identité sociale à celle de la productivité dans le cadre de la diffusion des districts marshalliens.

La troisième phase consiste donc à rechercher cette structure sociale dans le but de classer les systèmes locaux selon les caractéristiques de l'unité productive; et ce, de façon à pouvoir sélectionner les systèmes ayant les caractéristiques dominantes de cette structure avec celles «typiques» du district marshallien. Les résultats, et ainsi la conclusion de cette phase analytique, sont résumés dans les figures 6 et 7 (p. 176 et 177). Alors que les figures 1 et 2 montraient l'importance quantitative des districts industriels marshalliens en référence à l'industrie manufacturière dominante, les deux dernières figures présentent les résultats de l'occupation industrielle et des services pour l'Italie dans son ensemble.

Comme première réaction face à ces résultats, on peut être surpris de ne retrouver qu'un nombre relativement restreint de systèmes locaux en tant que districts industriels marshalliens possédant les caractéristiques sociales; ceci réduit le nombre de systèmes de productions spécialisées auxquels on pouvait s'attendre en tant que districts industriels marshalliens.

En effet, avec cette méthode, on n'a pu retenir comme district marshallien, par exemple, les systèmes locaux de Biella (textile) au Piemont, de Cantù (meuble) et de Vigevano (chaussures) en Lombardie, de Solofra (tanneries) en Campanie, même s'ils avaient des productions spécialisées. Ces systèmes locaux n'ont pas toutes les caractéristiques typiques recherchées; ils occupent donc une position intermédiaire à l'intérieur du modèle du système productif italien (Sforzi et Montagnini, 1986). En particulier, leur système de production est dominé par de grandes et moyennes entreprises sur leur territoire et par l'importance du secteur tertiaire. Même si la production industrielle est spécialisée et interdépendante et même si la main-d'oeuvre féminine est importante, ces structures diffèrent car elles ne sont pas basées sur les petits entrepreneurs et sur une typologie familiale afférente (Sforzi, 1986).

développement des compétences, des connaissances et des équipements : une telle «intégration», qui permet des liens grandissants et qui consolide les relations entre des parties séparées des organisations industrielles, se démontre par elle-même, par exemple avec une sécurité croissante dans le crédit commercial et avec des moyens et des habitudes de communication...» Ainsi s'exprime Marshall (1920, p. 200-201) au sujet du rapport traitant de la différenciation et de la spécialisation dans l'organisation industrielles. 
Fig. 4 Configuration spatiale des systèmes locaux en Italie, 1981

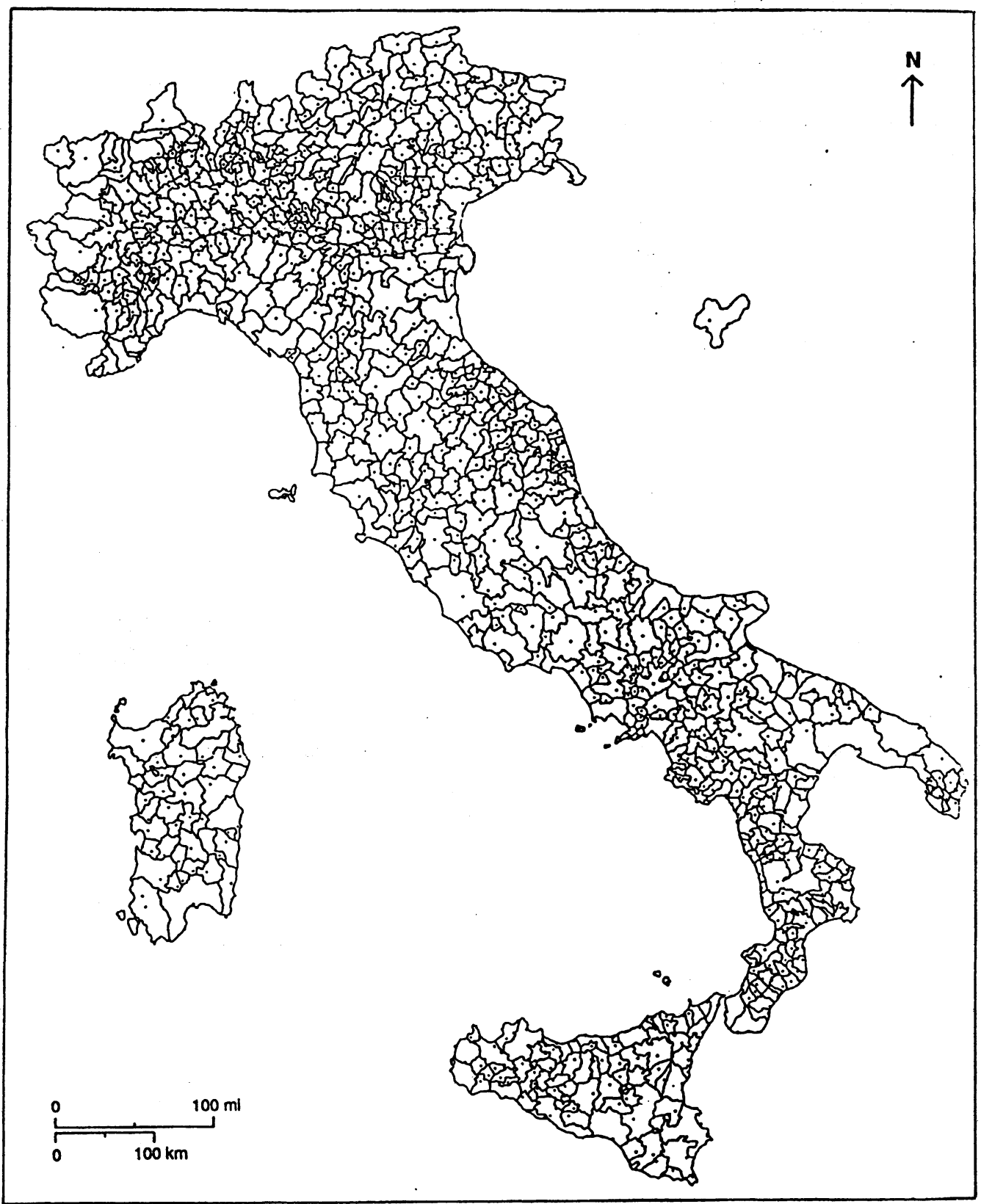


Fig. 5 Caractérisation sociale typique des districts industriels

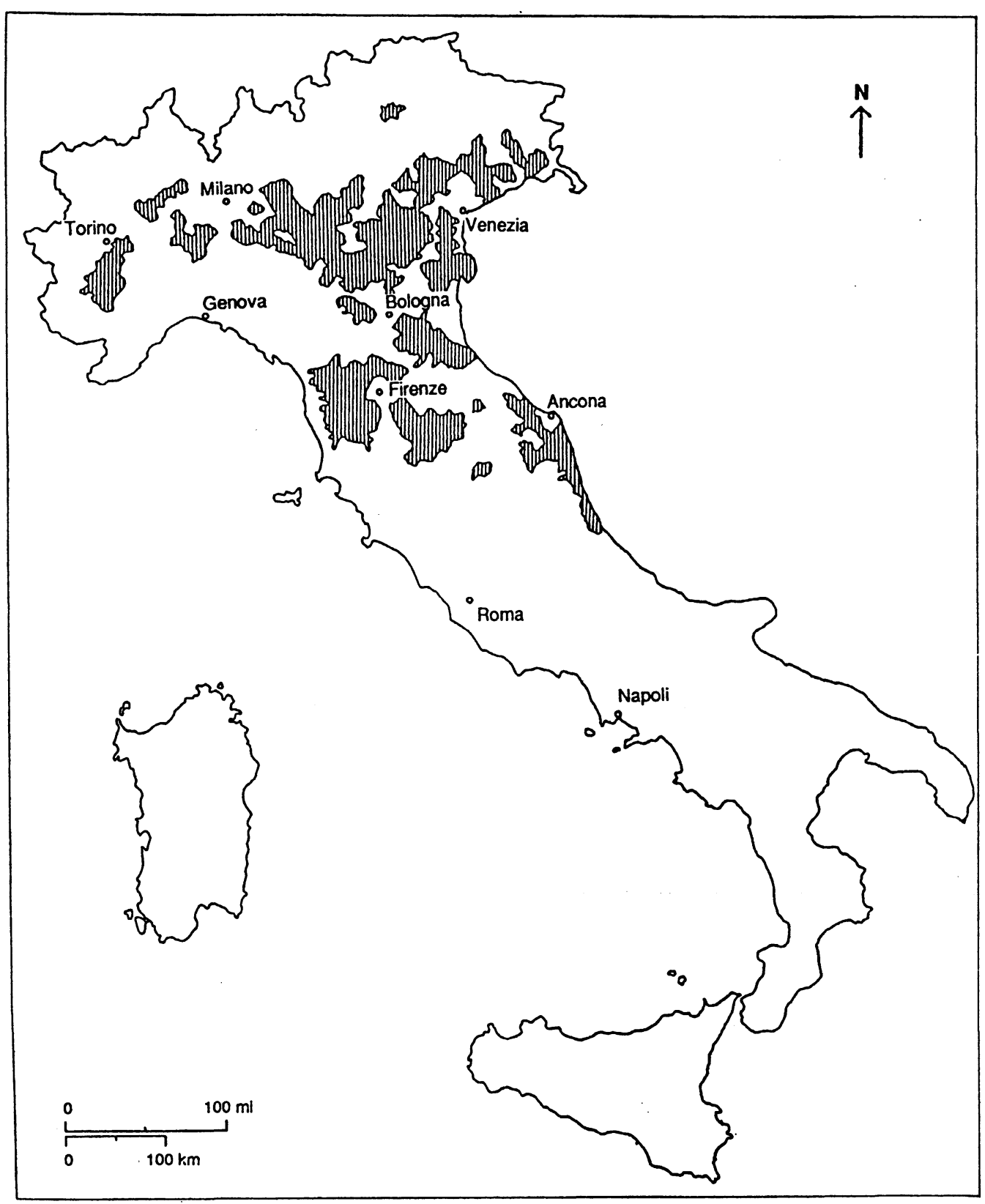


Fig. 6 Les districts industriels marshalliens

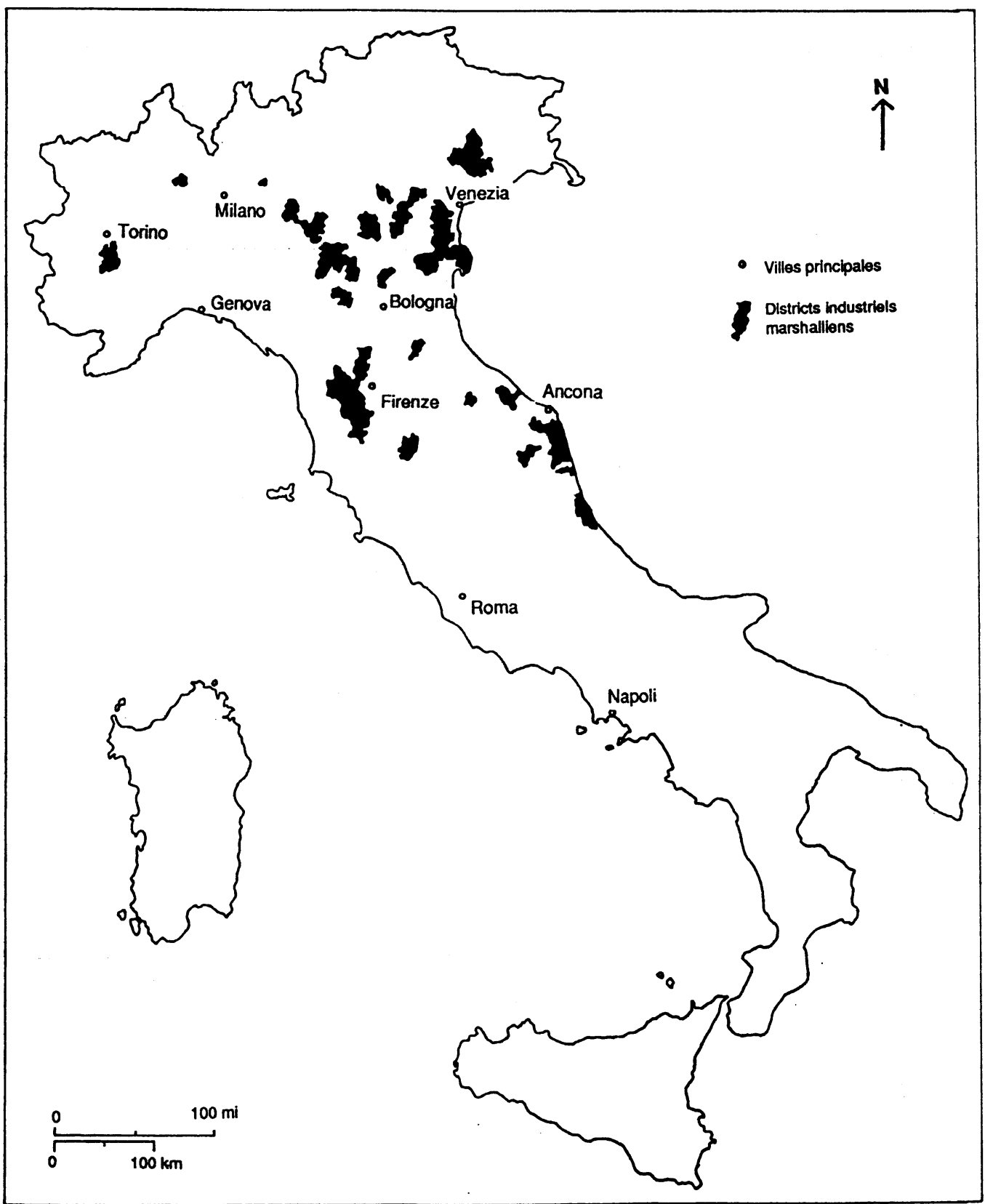


Fig. 7 Les districts industriels marshalliens selon leur industrie manufacturière dominante

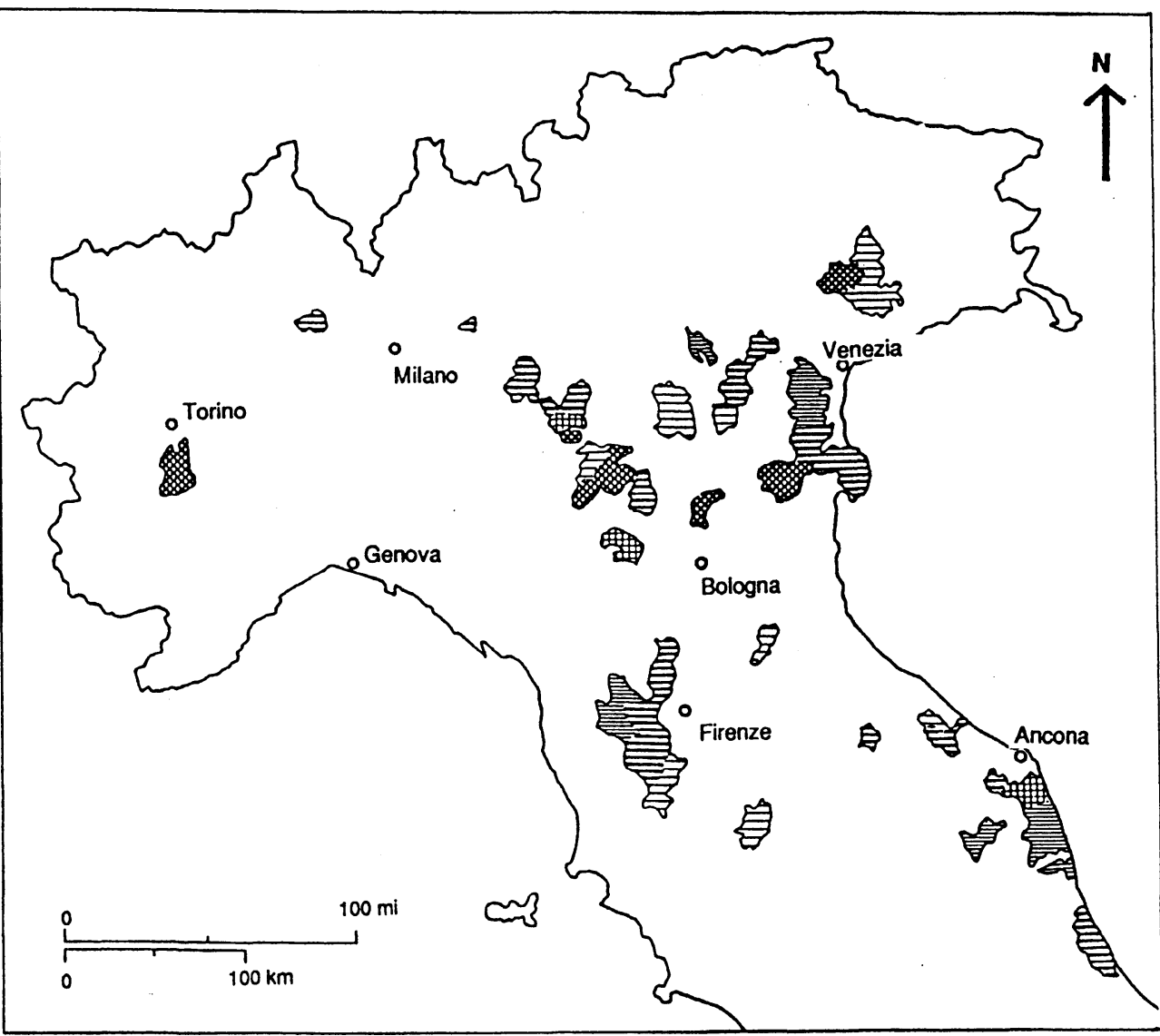

Equipements mécaniques et électro-mécaniques/ machinerie

- Villes principales

Textile/Habillement

Tannerie/articles en cuir/chaussure 
En fait, pour être reconnu comme district industriel marshallien, il faut avoir une structure sociale ayant les caractéristiques de ceux-ci, mais aussi une spécialisation au niveau de la production. Ainsi, les zones de Biella et de Cantù ne peuvent être reconnues comme districts, car leur structure sociale est différente; alors que les systèmes locaux de Faenza en Emilie-Romanie et d'Arezzo en Toscane, par exemple, auraient la structure sociale adéquate sans avoir une structure de production spécialisée telle que définie précédemment.

Toutefois, cette concentration et cette spécialisation relative du processus productif dans les systèmes locaux, valant tant au niveau national que local, amènent d'autres questions sur lesquelles il est nécessaire de s'arrêter, puisqu'une telle démarche d'identification de la réalité concrète peut comporter inévitablement de l'arbitraire, à cause des limites des données statistiques et de la subjectivité du processus analytique utilisé.

Trois points doivent être ajoutés pour résoudre ce problème. Deux de ces points relèvent de la procédure de recherche, alors que le troisième, plus ardu, porte sur l'interprétation du phénomène investigué. Une partie de l'explication peut venir de la dimension de la zone retenue regroupant telle structure sociale et productive. Les systèmes locaux retenus constituent, en effet, des «entités» définies plus par leurs relations entre leurs éléments productifs et ceux de l'habitat que par les caractéristiques (sociales et de production) très hétérogènes qui influencent ces derniers. Il ne peut en être autrement, puisque la mesure des caractéristiques ne touche que «des parties d'un tout» et non des «parties isolées». On comprend que l'on ne peut connaître les propriétés (les caractéristiques) d'un tout, d'une part, en mesurant isolément ses parties (chaque localité) ou en ignorant ce qu'est le tout (le système de localités interagissant) auxquelles elles appartiennent, ou encore, $d$ 'autre part, en retenant un ensemble qui ne relève ni de l'approche conceptuelle ni de l'analyse empirique.

Par exemple, l'industrie du meuble de Ponsacco en Toscane (qui constitue une localité du système local du Val d'Era) apparaît sous une lumière différente selon qu'on l'isole ou non de ce système local, ou encore qu'on mesure ses caractéristiques productive indépendamment du système local considéré dans la totalité. Ainsi, on peut dire que l'industrie du meuble constitue une caractéristique structurelle typique de la localité de Ponsacco, alors que ce n'est pas le cas du système local du Val d'Era.

Une autre explication touchant encore la démarche de recherche vient également du raisonnement concernant la présence, dans un système local, d'une réalité «équipotentielle» dans le processus de spécialisation de la production, sans que cette spécialisation ressorte de manière particulièrement évidente et même si celle-ci constitue la caractéristique typique du regroupement des entreprises appartenant au système local.

Cette ensemble de considérations sur la connaissance de la réalité concrète du district industriel marshallien pose le problème de la transformation des «complexes organisés» auxquels il est assimilable, quand ce district peut s'identifier avec 
l'entité économico-sociale localisée dans le temps. La réponse ne peut provenir de la vérification expérimentale, mais exige une réflexion ad hoc. Au-delà de l'analyse empirique, pour bien comprendre les phénomènes qui nous occupent et avant de recourir à des analyses empiriques plus récentes et plus fines, il faut passer par une nouvelle phase de réflexion théorique. Celle-ci, à l'état actuel d'ébauche, ne pourra qu'être faite en des termes généraux.

Ainsi, quand nous parlons du «processus de transformation des complexes organisés»,nous faisons référence à leur mode d'organisation et de développement pour passer d'un «stade initial équipotentiel» à une situation dans laquelle on a de la peine à reconnaître une activité comme dominante selon le stade de différenciation progressive de la structure originale. C'est-à-dire que le système passe d'une industrialisation multiple à une situation où un secteur industriel prend le pas sur les autres de façon à toujours plus influencer le comportement productif du complexe et à orienter toute la structure économique et sociale. En terme systémique, cette transition implique une augmentation de la complexité interne du système; celle-ci tend à évoluer vers un ordre plus élevé qui se développe de façon interactive avec les autres systèmes de l'environnement circonstantiel selon un processus d'allocation/ réallocation d'activités donnant lieu à une nouvelle configuration structurelle (Bertafanffy 1971).

\section{Dernières considérations pour conclure : le stade de développement du district industriel marshallien}

En fait, on peut voir les systèmes territoriaux locaux comme une entité qui s'organise pour se différencier progressivement et évoluer d'un état de faible complexité à une complexité plus élevée. Mais le fait de devenir plus spécialisé a des implications directes sur l'obtention des conditions d'évolution vers un district industriel marshallien. Il est vrai qu'un organisme économico-social spatialisé localement demeure toujours un système unitaire même si certaines parties prennent progressivement le pas sur d'autres. Dans cette évolution, le passage d'une indifférenciation globale à une spécialisation de ses parties peut entraîner la dissolution de tout le système. Plus les parties deviennent spécialisées, plus la cohérence devient fragile. Ce qui conduit à une issue "catastrophique» pour le système complexe. En effet, l'incapacité du système d'auto-régulariser la décroissance ou la croissance et la diversification-spécialisation interne augmente sa dépendance relative aux interactions avec l'environnement externe. Le cas du district industriel marshallien représente justement un «état de spécialisation progressive» selon un processus à la base de la formation du modèle d'industrialisation légère; et cet état dépend en grande partie de ses interactions avec l'environnement et avec les autres systèmes territoriaux selon leur différent stade de développement. 
Les districts industriels marshalliens en Italie :

À la lumière des connaissances empiriques disponibles et de la réflexion théorique, il n'est pas possible actuellement d'apporter une réponse claire sur l'état actuel du district industriel marshallien. Sa stabilité dépend des possibilités de «conflits» et du mode de réponse qu'il développe face à l'évolution de son environnement.

\section{BIBLIOGRAPHIE}

Bagnasco, A., (1977), Tre Italie. La problematica territoriale dello sviluppo italiano, Il Mulino, Bologne.

Becattini, G., (1978), «The development of light industry in Tuscany : an interpretation», Economic Notes, 2-3.

Becattini, G., (1979), «Dal settore industriale al distretto industriale. Alcune considerazioni sull'unità d'indagine dell'economia industriale», Rivista di Economia e Politica Industrialel. [reproduit de : «L'unità d'indagine», dans G. Becattini, (éd.), Mercato e forze locali : il distretto industriale, Il Mulino, Bologne].

Becattini, G., (1989), «Riflessioni sul distretto industriale marshalliano come concetto socioeconomico», State e Mercato, 1.

Bellandi, M., (1982), «Il distretto industriale in Alfred Marshall», L'Industria, 3. [reproduit de : «La formulazione originaria», dans G. Becattini, (éd.),Mercato e forze locali : il distretto industriale, II Mulino, Bologne].

Bertalanffy, L., (1971), Teoria generale dei sistemi, ISEDI, Milan.

Chapman, G.P., (1977), Human and Environmental Systems : A Geographer's Appraisal, Academic Press, London.

Fuà, G., (1983), «L'industrializzazione del Nord Est e Centro», dans G. Fuà et C. Zacchia, (éd.), Industrializzazione senza fratture, Il Mulino, Bologne.

Garofoli, G., (1983), «Le aree sistema in Italia», Politica ed Economia, 11.

Hägerstrand, T., (1970), «What about people in regional science?», Papers of the Regional Science Association, XXIV.

Harvey, D., (1969), Explanation in Geography, Amold, London.

Harvey, D., (1985), «The geopolitics of capitalism». dans D. Gregory et J. Urry, (éd.), Social Relations and Spatial Structures, Macmillan, London, 128-163.

IRPET, (1969), «Lo sviluppo economico della Toscana : un'ipotesi di lavoro», Florence.

IRPET, (1975), Lo sviluppo economico della Toscana, con particolate riguardo all' industrializzazione leggera, édité par G. Becattini, Guaraldi, Firenze.

ISTAT-IRPET,(1986a), Imercati locali del lavoro in Italia, Rome, [reproduit chez: F. Angeli, Milan, 1989].

ISTAT-IRPET, (1986b), «Le zone residenziali in Italia», Rome.

ISTAT-IRPET, (1986c), «Le zone produttive in Italia», Rome.

Jonas, A., (1988), «A new regional geography of localities?», Area, 2.

King, R., (1985), The Industrial Geography of Italy, Croom Helm, London.

Marshall, A., (1920), Principles of Economics, Huitième Edition, Macmillan, London.

Openshaw, S. et Sforzi, F., (1983), «Metodologia per l'analisi della struttura sociale urbana», IRPET, Florence. 
Sforzi, F., (1982), «Identificazione degli ambiti sub-regionali di programmazione», dans M. Bielli e A. La Bella, (éd.), Problematiche dei livelli sub-regionali di programmazione, F. Angeli, Milan.

Sforzi, F., (1986), «L'Italia marginale : una valutazione geografica», communication présentée à la Vll conférence italienne de Science Régionale, Urbino, 14-17 septembre [reproduit dans : A. Becchi Collidà, E. Ciciotti et A. Mela, (éd.), Aree interne, tutela del territorio e valorizzazione delle risorse, F. Angeli, Milan, 1989].

Sforzi, F., (1987), «Mercati locali-regionali del lavoro : problemi di definizione ed evidenza empirica in Italia», dans T. Gallino, G. A. Rabino et F. Viano, (éd.), L'articolazione territoriale dei mercati del lavoro, IRES, Turin.

Sforzi, F.et M. Montagnini, (1986), I sistemi produttivi locali in Italia, IRPET, Florence.

Sforzi, F., Openshaw, S. et Wymer, C., (1982), «La delimitazione di sistemi sub-regionali : scopi algoritmi, applicazioni», IRPET, Florence.

Sforzi, F., Openshaw, S. et Wymer, C., (1983), «Le aree sociali della Toscana», IRPET, Florence.

Sforzi, F., Openshaw, S. et Wymer, C., (1984), «L'organizzazione dello spazio produttivo regionale : metodi e applicazioni», IRPET, Florence. 
Appendice

\section{Légende de la figure 7}

Mécanique : $\quad$ Carmagnola (Piemont), Rivarolo Mantovano (Lombardie), Sant'Ilario d'Enza (Emilie-Romanie)

Machinerie : $\quad$ Suzzara (Lombardie), Novellara (Emilie-Romanie) Cento (Emilie-Romanie), Copparo (Emilie-Romanie)

Electro-mécanique : Conegliano (Veneto), Guastalla (Emilie-Romanie)

Textile :

Urgnano (Lombardie), Quinzano d'Oglio (Lombardie), Asola (Lombardie), Carpi (Emilie-Romagnie), Prato (Toscane)

Habillement : $\quad$ Oleggio (Piemont), Manerbio (Lombardie), Pontevico (Lombardie), Verolanuova (Lombardie), Ostiano (Lombardie), Noventa Vicentina (Veneto), Piazzola sul Brenta (Veneto), Adria (Veneto), Porto Tolle (Veneto), Mondolfo (Marche), Urbania (Marche), Corinaldo (Marche), Filottrano (Marche), Roseto degli Abruzzi (Marche), Castelfiorentino (Toscane), Empoli (Toscane)

Tannerie :

Arzignano (Veneto), Santa Croce sull'Arno (Toscane)

Articles en cuir : $\quad$ Tolentino (Marche)

Chaussures :

San Giovanni Ilarione (Veneto), Piove di Sacco (Veneto), Civitanova Marche (Marche), Fermo (Marche), Grottazzolina (Marche), Montefiore dell'Aso (Marche), Montegranaro(Marche), Monte San Pietrangeli (Marche), Torre San Patrizio (Marche), Lamporecchio (Toscane), Montecatini Terme (Toscana)

Meubles :

Viadana (Lombardie), Bovolone (Veneto), Cerea (Veneto), Nogara (Veneto), Motta di Livenza (Veneto), Oderzo (Veneto), Montagnana (Veneto), Sacile (Friuli-Venezia Giulia), Modigliana (Emilie-Romanie), Saltara (Marche), Poggibonsi (Toscane), Sinalunga (Toscane)

Céramique : $\quad$ Sassuolo (Emilie-Romanie), Casalgrande (Emilie-Romanie

Jouets : $\quad$ Canneto sull'Oglio (Lombardie)

Instruments musicaux : Potenza Picena (Marche), Recanati (Marche) 
Tab. 1 Les districts industriels marshalliens selon leur Industrie manufacturière dominante en 1981

\begin{tabular}{|c|c|c|c|c|c|c|}
\hline $\begin{array}{l}\text { District } \\
\text { selon l'industrie } \\
\text { manufacturière dominante }\end{array}$ & $\begin{array}{l}\text { Nombre } \\
\text { de } \\
\text { Districts }\end{array}$ & $\begin{array}{l}\text { Emploi dans } \\
\text { dans l'industrie } \\
\text { manufacturière } \\
\text { dominante } \\
\text { (a) }\end{array}$ & $\begin{array}{c}\text { Emploi dans } \\
\text { toute l'industrie } \\
\text { manufacturière } \\
\text { (b) }\end{array}$ & $\begin{array}{l}\text { Emploi } \\
\text { au total } \\
\text { (c) }\end{array}$ & $\begin{array}{c}\text { \% d'emploi } \\
\text { dans l'industrie } \\
\text { manufacturière } \\
\text { dominante } \\
(a / b)\end{array}$ & $\begin{array}{c}\text { \% d'emploi } \\
\text { dans toute l'industrie } \\
\text { manufacturière } \\
\text { (b/c) }\end{array}$ \\
\hline Mécanique & 3 & 8.005 & 23.370 & 42.037 & 34,3 & 55,6 \\
\hline Machinerie & 4 & 10.973 & 28.915 & 52.665 & 37,9 & 54,9 \\
\hline Électromécanique & 3 & 5.826 & 23.642 & 45.662 & 24,6 & 51,8 \\
\hline Textile & 5 & 68.593 & 93.103 & 156.070 & 73,7 & 59,7 \\
\hline Habillement & 16 & 29.962 & 88.173 & 173.373 & 34,0 & 50,9 \\
\hline Tannerie & 2 & 13.721 & 35.586 & 56.097 & 38,6 & 63,4 \\
\hline Chaussure & 11 & 51.303 & 80.592 & 149.986 & 63,7 & 53,7 \\
\hline Articles en cuir & 1 & 1.318 & 3.835 & 8.083 & 34,4 & 47,4 \\
\hline Meuble & 12 & 31.641 & 66.192 & 131.274 & 47,8 & 50,4 \\
\hline Céramique & 2 & 22.418 & 36.856 & 59.563 & 60,8 & 61,9 \\
\hline Instrument de musique & 2 & 4.162 & 13.541 & 25.664 & 30,7 & 52,8 \\
\hline Jouets & 1 & 972 & 2.800 & 5.535 & 34,7 & 50,6 \\
\hline TOTAL & 61 & 248.894 & 496.605 & 906.009 & 50,1 & 54,8 \\
\hline
\end{tabular}

Source: Données calculées à partir de ISTAT, 6 recensement général de l'industrie, du commerce, des services et de l'artisanat, 26 octobre 1981. 
Tab. 2 Emploi dans l'industrie manufacturière et les services des districts industriels marshalliens (DIM) et en Italie, 1981

\begin{tabular}{|c|c|c|c|c|}
\hline Activités économiques & DIM & $\begin{array}{l}\text { ITALIE } \\
\text { (b) }\end{array}$ & $\begin{array}{c}\% \\
(a / b)\end{array}$ & $\begin{array}{l}\text { LQ } \\
\left({ }^{\star}\right)\end{array}$ \\
\hline Agriculture & 13.066 & 175.080 & 7,5 & 1,39 \\
\hline Mines & 1.483 & 55.149 & 2,7 & 0,50 \\
\hline Industrie manufacturière & 496.605 & 5.763 .988 & 8,6 & 1,61 \\
\hline $\begin{array}{l}\text { Transformation métallique et produits dérivés, et industrie chimique } \\
\text { Industrie des produits métalliques et mécaniques de précision }\end{array}$ & 106.253 & 2.221 .233 & 4,8 & 0,89 \\
\hline Autres industries manufacturières & 340.210 & 2.701 .407 & 12,6 & 2,35 \\
\hline Construction & 69.753 & 1.192 .398 & 5,8 & 1,09 \\
\hline Commerce, publicité, hébergement; réparation & 146.960 & 3.751 .146 & 3,9 & 0,73 \\
\hline Transport et communication & 29.940 & 1.148 .489 & 2,6 & 0,49 \\
\hline \multirow{2}{*}{$\begin{array}{l}\text { Habitants } \\
\text { Taux d'emploi }\end{array}$} & 2.435 .211 & 56.335 .678 & 4,3 & \\
\hline & 37,2 & 30,0 & & \\
\hline \multicolumn{5}{|c|}{$\begin{array}{l}\text { (") Le coefficient de concentration (LQ) est défini comme: (Oij/Oi) / (Aj/N) } \\
\text { où } \mathrm{Oij}=\text { emploi dans l'industrie i de la zone j } \\
\begin{aligned} \mathrm{O} i & =\text { emploi national de l'industrie } \mathrm{i} \\
\mathrm{Aj} & =\text { emploi total de la zone } \mathrm{j} \\
\mathrm{N} & =\text { emploi national total }\end{aligned}\end{array}$} \\
\hline
\end{tabular}

Source:Données calculées à partir de ISTAT, 6ième recensement générale de l'industrie, du commerce, des services et de l'artisanat, 26 octobre 1981. 